\section{G392(P) PREPARING FOR PAEDIATRIC INTENSIVE CARE - THE DEVELOPMENT OF A PAEDIATRIC INTENSIVE CARE UNIT SIMULATION INDUCTION COURSE FOR TRAINEE DOCTORS}

L Blakemore, J Lumsden. Paediatric Intensive Care Unit, Leeds Teaching Hospitals Trust, Leeds, UK

\subsection{6/archdischild-2015-308599.346}

Aims On our paediatric intensive care unit (PICU) doctors induction traditionally consisted of a series of lectures and small group work. In order to make introduction more interactive and enhance learning, we decided to set up a one day simulation based induction course.

Methods Learning needs were identified from informal survey of current trainees and PICU consultants. Four simulation scenarios were designed to encompass the learning objectives established from the learning needs survey and key competencies needed for PICU. Scenarios involved patients transferred to PICU for stabilisation, or deteriorating patients on the unit. 30 min simulations and were followed by $1 \mathrm{~h}$ debrief and tutorial. Tutorials focused on medical management and practicalities such how to set up a ventilator or give inotropes. Simulations ran with 2-3 trainees and 2 PICU nurses per scenario, and were set up in a cubicle with standard equipment, using high fidelity manikins. Due to anticipated inexperience, trainees were offered "time out" and "ask the audience" options during the scenarios to enable them to draw on the knowledge of their peers.

Results 10 trainees completed the simulated induction course. When asked to use a 5 point likert scale, $90-100 \%$ of trainees either agreed or strongly agreed to all but one of the 9 statements related to the course meeting learning objectives and knowledge needs. High frequency oscillation was the single area that was identified by trainees as not being covered in desired depth. Free text feedback was overall very positive. Suggestions for improvement would be to provide a specific ventilation workshop to enable this subject to be covered in greater depth, plus a written handout to supplement the day. We are waiting to see how the induction evaluates in the GMC survey, compared to traditional induction.

Conclusion We ran a pilot PICU simulation induction which was well received by trainees, and met all but one learning objective. The course could be easily adapted to address this objective and could be tried in other Paediatric Intensive Care Units.

\section{G393(P) REDUCTION IN PRESCRIPTION ERRORS IN A NEONATAL INTENSIVE CARE UNIT: A COMPLETED AUDIT CYCLE}

${ }^{1} \mathrm{~T}$ Fordham, ${ }^{1} \mathrm{H}$ Green, ${ }^{2} \mathrm{Q}$ Badeaa, ${ }^{2} \mathrm{H}$ Ibrahim, ${ }^{2} \mathrm{NV}$ Subhedar. 'School of Medicine, University of Liverpool, Liverpool, UK; ${ }^{2}$ Neonatal Unit, Liverpool Women's Hospital, Liverpool, UK

\subsection{6/archdischild-2015-308599.347}

Background Neonates are particularly vulnerable to harm from medication-related errors. Prescription errors are one of the most important sources of potential harm accounting for approximately two-thirds of all medication-related incidents in this population.

Aims To evaluate the impact of a package of interventions designed to improve prescribing practice by doctors and advanced neonatal practitioners (ANNPs) working in a large UK NICU.
Methods We devised an audit tool to capture data relating to the quality of prescribing across a range of neonatal drug and intravenous infusion/parenteral nutrition prescriptions. The accuracy and completeness of prescriptions were assessed against a list of agreed standards. Prescription charts were selected at random, weekly on the same day each week over a seven week period in both audits; a single individual undertook the baseline audit whereas two individuals performed the re-audit. Following the baseline audit a number of interventions were introduced and implemented including modification of prescription charts, specific improvements in education and training and anonymised publication of prescribers' error rates. Prescribing practice was re-assessed one year later after the package of interventions had been embedded into routine practice.

Results 1087 individual prescriptions were reviewed in total. During the initial audit, there were 16 errors in a total 292 prescriptions assessed giving an error rate of 5.5 per 100 prescriptions. In the re-audit, there were a total of 13 errors in 795 prescriptions examined giving an error rate of 1.64 errors per 100 prescriptions ( $p=0.003$ compared with the baseline audit). All 13 observed errors were deemed relatively minor prescribing errors and none led to any patient harm. Prescribers were not identifiable in 126 prescriptions (16\%).

Conclusions Prescribing errors in neonatal practice are relatively common but rarely result in patient harm. Using a completed audit cycle, we have shown a reduction in prescribing error rates following the implementation of a range of interventions that combined to improve prescribing practice of junior doctors and ANNPs.

\section{G394(P) INFECTION AND WHEEZE IN THE FIRST 6 MONTHS OF LIFE - AN INTERIM ANALYSIS OF THE GO-CHILD BIRTH COHORT}

${ }^{1} \mathrm{~K}$ Basu, ${ }^{1} \mathrm{~K}$ Fidler, ${ }^{1} \mathrm{AJ}$ Abd, ${ }^{2} \mathrm{SK}$ Inglis, ${ }^{3} \mathrm{M}$ Henry, ${ }^{4} \mathrm{~A}$ Memon, ${ }^{1} \mathrm{H}$ Rabe, ${ }^{1} \mathrm{P}$ Seddon, ${ }^{5} \mathrm{R}$ Tavendale, ${ }^{5} \mathrm{CNA}$ Palmer, 'S Mukhopadhyay. 'Academic Department of Paediatrics, Royal Alexandra Children's Hospital, Brighton and Sussex Medical School, Brighton, UK; ${ }^{2}$ Tayside Clinical Trials Unit and Maternal and Child Health Sciences, Ninewells Hospital and Medical School, University of Dundee, Dundee, UK; ${ }^{3}$ College of Medical and Dental Sciences, University of Birmingham, Birmingham, UK; ${ }^{4}$ Division of Primary Care and Public Health, University of Brighton, Brighton, UK; ${ }^{5}$ Biomedical Research Institute, Ninewells Hospital and Medical School, University of Dundee, Dundee, UK

\subsection{6/archdischild-2015-308599.348}

Aims The aim of the GO-CHILD birth cohort study is to understand the natural history of common childhood diseases like asthma, allergies and infections and to investigate the role of gene-environmental interactions in childhood diseases that present very early in life. We wish to understand how these associations during $0-2$ years relate to our previous observations on older children. We report our interim observations regarding disease phenotype at 6 months of age.

Methods GO-CHILD is a multicentre prospective birth cohort study. 2315 infants were recruited from antenatal clinics across the UK and followed up at the ages of 3, 6, 9, 12 and 24 months through postal questionnaires. The 6-month questionnaire explores infection and atopy related events in the first 6 months of life.

Results 938 participants returned the completed 6-month questionnaire. $106(11 \%)$ infants had been admitted to the special care baby unit, of whom $41 \%$ had confirmed or suspected infections and $40 \%$ reported breathing problems. 130 (13.8\%) children reported wheeze in the first 6 months of life. Among them, 